OPEN

SUBJECT AREAS:

LONG-TERM

POTENTIATION

ALZHEIMER'S DISEASE

Received

15 August 2014

Accepted

31 October 2014

Published

2 December 2014

Correspondence and requests for materials should be addressed to O.A. (oal@columbia.

edu)

\section{Regulation of synaptic plasticity and cognition by SUMO in normal physiology and Alzheimer's disease}

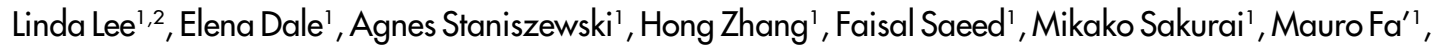
Ian Orozco', Francesco Michelassi' ', Nsikan Akpan', Helaina Lehrer ${ }^{3}$ \& Ottavio Arancio

${ }^{1}$ Taub Institute and Department of Pathology and Cell Biology, Columbia University, New York, NY 10032, USA, ${ }^{2}$ Department of Neurobiology and Behavior, Columbia University, New York, NY 10032, USA, ${ }^{3}$ Department of Biochemistry and Molecular Biophysics, Columbia University, New York, NY 10032, USA.

Learning and memory and the underlying cellular correlate, long-term synaptic plasticity, involve regulation by posttranslational modifications (PTMs). Here we demonstrate that conjugation with the small ubiquitin-like modifier (SUMO) is a novel PTM required for normal synaptic and cognitive functioning. Acute inhibition of SUMOylation impairs long-term potentiation (LTP) and hippocampal-dependent learning. Since Alzheimer's disease (AD) prominently features both synaptic and PTM dysregulation, we investigated SUMOylation under pathology induced by amyloid- $\beta(A \beta)$, a primary neurotoxic molecule implicated in AD. We observed that SUMOylation is dysregulated in both human AD brain tissue and the Tg2576 transgenic AD mouse model. While neuronal activation normally induced upregulation of SUMOylation, this effect was impaired by $A \boldsymbol{\beta}_{42}$ oligomers. However, supplementing SUMOylation via transduction of its conjugating enzyme, Ubc9, rescued A $\beta$-induced deficits in LTP and hippocampal-dependent learning and memory. Our data establish SUMO as a novel regulator of LTP and hippocampal-dependent cognition and additionally implicate SUMOylation impairments in AD pathogenesis.

$\mathrm{P}$ osttranslational modifications (PTMs) are ubiquitously involved in cell signaling cascades. Such modifications allow for the rapid and highly dynamic modulation of a cell's signaling networks and its responses to the environment. In the nervous system, regulation by PTMs is of critical necessity for complex neuronal processing and is a well-established general mechanism required for learning and memory as well as the underlying cellular correlate, long-term synaptic plasticity ${ }^{1-4}$.

The regulation of PTMs can become disrupted and dysfunctional under pathological conditions. In AD, as well as other neurodegenerative diseases, abnormal phosphorylation and ubiquitination are pathological hallmarks $\mathrm{s}^{5,6}$. Aberrant PTM regulation can occur cell-wide as well as in the localized microenvironments of synapses. For example, one of the mechanisms by which $\mathrm{A} \beta$, a primary molecular culprit in $\mathrm{AD}$, impairs synaptic transmission is through the dysregulation of neurotransmitter receptor phosphorylation ${ }^{7,8}$. The disruption of normal PTMbased signaling at the synapse is a pathological mechanism that likely contributes to cognitive dysfunction in diseases such as AD.

Recently, another PTM - the small ubiquitin-like modifier (SUMO) - has been described for multiple neuronal proteins. SUMOylation involves the covalent attachment of an $11 \mathrm{kDa}$ SUMO protein to a lysine residue on the target. There are three known SUMO paralogs in vertebrate brains: SUMO1-3. Since SUMO2 and SUMO3 share $\sim 95 \%$ sequence homology and have not been differentiated functionally, they are often collectively referred to as SUMO2 $/ 3^{10,11}$. SUMO1 and SUMO2/3 are expressed abundantly in the adult brain ${ }^{12,13}$. In addition, the sole E2type conjugating enzyme for all SUMO paralogs, Ubc9, is expressed throughout the cerebral cortex and hippocampus, with particularly high levels in dentate granule cells and pyramidal neurons ${ }^{14}$. Modification by SUMO can alter multiple functional properties of the target protein including localization, activity and protein-protein interactions $s^{10}$.

Since its discovery, SUMOylation has been best-characterized for its roles in nuclear and genomic maintenance $^{11}$. Recently, the involvement of SUMOylation in extranuclear neuronal functioning and neurological diseases has been gaining traction. Several proteins with neuron-specific roles are now known to be SUMOylated, including transcription factors and neurotransmitter receptors ${ }^{15}$. However, while SUMOylation has been shown 
to be involved in a specific type of basal synaptic transmission ${ }^{16}$, the role of SUMOylation in long-term potentiation (LTP) and cognition is unknown. Furthermore, given its role in synaptic functioning, the potential involvement of SUMOylation in the cognitive impairment that characterizes $\mathrm{AD}$ constitutes an unanswered question with potential therapeutic implications.

To address these issues, we asked three main questions. First, is SUMOylation involved in and required for normal synaptic plasticity and cognition? Second, is SUMOylation impacted by A $\beta$-related pathology, and is there dysregulation of this PTM in human AD brain and AD mouse models? And third, can detected SUMOylation changes be countered to improve synaptic and cognitive functioning? By examining global SUMO conjugation levels as an assay for changes in its regulation, we discovered that SUMOylation is dynamically regulated by neuronal activation. Furthermore, acute inhibition experiments demonstrated that SUMOylation is indeed required for both normal LTP as well as hippocampal-dependent learning and memory. In investigating potential alterations of SUMOylation with AD pathology, we discovered that activitydependent SUMOylation is impaired by both acutely and chronically elevated levels of $A \beta$ peptides. This impairment is evident as decreased levels of basal SUMOylation in a transgenic AD mouse model and human post-mortem AD hippocampi. Lastly, in order to determine the pathogenic relevance of this SUMO impairment, we enhanced SUMOylation via Ubc9 transduction and observed that deficits in LTP and hippocampal-dependent cognition were rescued. Our findings highlight SUMO as an important PTM involved in long-term synaptic plasticity and furthermore implicate dysregulated SUMOylation in the cognitive deficits that characterize AD. This latter result indicates that targeting the SUMO system could be a novel drug discovery avenue for $\mathrm{AD}$ as well as other neurological diseases featuring cognitive impairment.

\section{Results}

Neuronal depolarization regulates SUMOylation. In synaptosomal preparations, SUMOylation was found to be involved in presynaptic functioning ${ }^{17}$. However, it was unknown if dynamic SUMO regulation occurs with synaptic activity and plasticity in intact brain tissue. To investigate this issue, we used an activating stimulation to study if and how SUMOylation is regulated in situ. With acute hippocampal slices, we used a two minute incubation in elevated $\mathrm{KCl}(50 \mathrm{mM})$ buffer to briefly depolarize and stimulate neurons. This type of highpotassium protocol results in a type of long-term potentiation (LTP) in slices that shares several characteristics with tetanus-induced $\mathrm{LTP}^{18,19}$. Slices were collected immediately after stimulation or following several incubation times in ACSF buffer. At 5 minutes post-stimulation, there was a $40-50 \%$ increase in SUMOylation, compared to non-stimulated controls, that was primarily evident in the high molecular weight region of SUMO conjugates $(\sim 150$ $350 \mathrm{kD}$ ) (Fig. 1a).

This upregulation of high molecular weight conjugates is consistent with previous studies that have observed a similar SUMO effect, wherein various manipulations cause SUMOylation changes that are primarily observed in the high molecular weight region ${ }^{20-22}$. Since SUMO2/3 can form polymeric SUMO chain modifications ${ }^{23}$, this region may include various poly-SUMOylated conjugates which are especially labile and likely to reflect changes in SUMO regulation. This lability is evident in the reversal of the global SUMOylation effect at 30 minutes post-depolarization (Fig. 1a). We observed that these changes are specific for SUMO2/3; no changes were observed in SUMO1 conjugation (Fig. S1a). These data indicate that hippocampal SUMO2/3 conjugation in situ can be dynamically regulated by neuronal activation.

To determine whether the upregulation resulted from the depolarization itself or from any evoked activity ${ }^{24,25}$, we further analyzed the SUMOylation increase at the 5 minute time point that showed the maximal effect. Tetrodotoxin (TTX), an inhibitor of voltage-gated sodium channels, was used to block action potentials for one hour before and during the stimulation and recovery times. When slices were treated with TTX $(1 \mu \mathrm{M})$, stimulation no longer resulted in increased SUMOylation (Fig. S1b). Thus, the high KCl-induced SUMOylation appears to be dependent on neuronal activity. It has been previously reported that a long TTX incubation ( 24 hours) in neuronal cultures leads to an increase in SUMO1 conjugation ${ }^{26}$; with our 1 hour TTX protocol in acute slices, we did not observe changes in basal non-stimulated global SUMOylation levels (Fig. S1c).

Demonstrating that activity-induced SUMOylation is not limited to high-potassium stimulation, we also found that SUMOylation increases occur with a tetanizing stimulus that is commonly used to induce LTP. In acute hippocampal slices, theta-burst stimulation resulted in significantly enhanced SUMOylation in area CA1, starting from 10 minutes post-tetanus and lasting at least one hour (Fig. S1d). While the time course of the elevated SUMOylation differs from that of high- $\mathrm{KCl}$ stimulation, likely reflecting differences in stimulation strength and coverage (whole slice vs. CA1), both sets of experiments support the dynamic activity-dependent regulation of SUMOylation in the hippocampus.

SUMOylation can be manipulated via transduction of Ubc9. In order to manipulate SUMOylation to study its involvement in synaptic plasticity, we developed new SUMO-targeted tools. To ensure high specificity, and since there were few readily-available SUMO-targeted small molecule compounds, we used the TAT protein transduction system. The HIV-1 TAT protein transduction domain has been previously used to successfully deliver a variety of functional proteins into cells ${ }^{27,28}$. To alter SUMOylation, we utilized Ubc9 which, as the sole SUMO conjugating enzyme, presents a way to globally and specifically manipulate SUMOylation. We made constructs with either the wild-type enzyme or a version with a dominant negative (DN) point mutation in which a catalytic cysteine is changed to a serine (C93S). This mutation has been previously described and used for dominant negative inhibition of overall SUMOylation ${ }^{29-31}$. The full-length enzymes were recombinantly fused to the TAT protein transduction domain (Fig. 1b). Wild-type TAT-Ubc9 exhibited SUMO conjugation functionality while the dominant negative TAT-Ubc9(DN) did not (Fig. 1c). The TAT-Ubc9 proteins were able to transduce hippocampal slices and primary neuron cultures (Fig. 1d, S2a, S2b).

When tested in slices stimulated with high potassium, application of wild-type TAT-Ubc9 trended towards enhanced SUMOylation following stimulation but this effect did not achieve statistical significance (Fig. 1e); this may indicate that there is a ceiling threshold on SUMOylation. However, this functional Ubc9 transduction enzyme would allow us to supplement SUMO conjugation capacity under conditions where SUMOylation is impaired. In contrast, application of dominant negative TAT-Ubc9(DN) was able to fully block the depolarization-induced increase in SUMOylation (Fig. 1e). Notably, the stimulation was necessary to fully utilize the transduced enzymes - under basal, non-stimulated conditions, neither TATUbc9 nor TAT-Ubc9(DN) significantly affected SUMO conjugate levels. These TAT transduction enzymes allowed us to specifically and acutely manipulate stimulus-dependent SUMOylation with relatively high temporal and spatial control.

SUMOylation is required for normal long-term potentiation. To date, although multiple neuronal SUMO substrates have been identified, the involvement of SUMOylation in long-term potentiation was unknown. With the development of the TATUbc9 transduction enzymes, we were able to investigate this issue by specifically inhibiting SUMOylation in acute hippocampal slices and observing the effects on LTP. When TAT-Ubc9(DN) (100 nM) was perfused onto slices for 1 hour prior to induction, LTP was significantly reduced compared to interleaved vehicle, TAT-Ubc9 
a
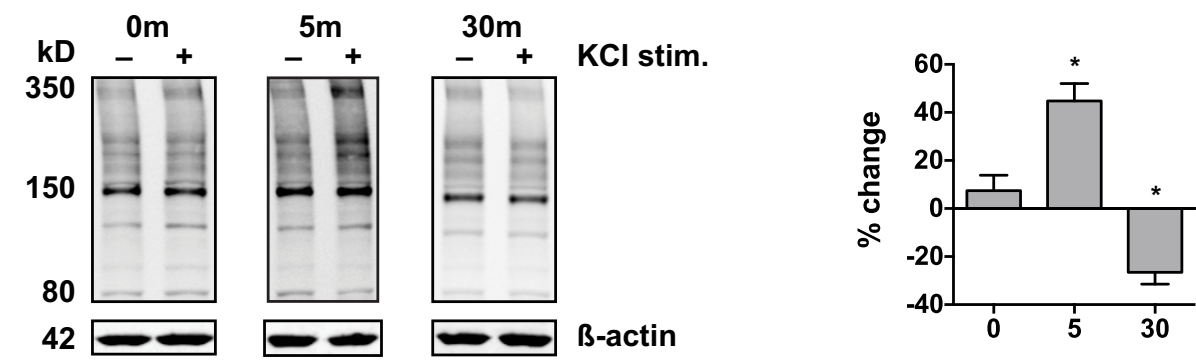

\section{Post-depolarization incubation (min)}

b

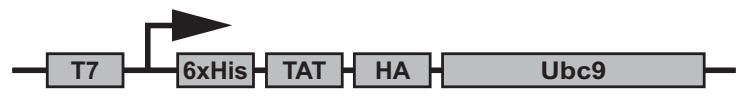

C ctl -ATP TAT DN d

TAT-Ubc9 (25 kD)

Ubc9 (18 kD)

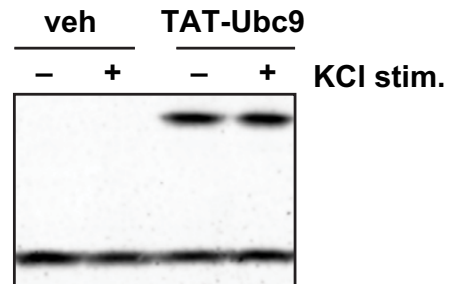

e

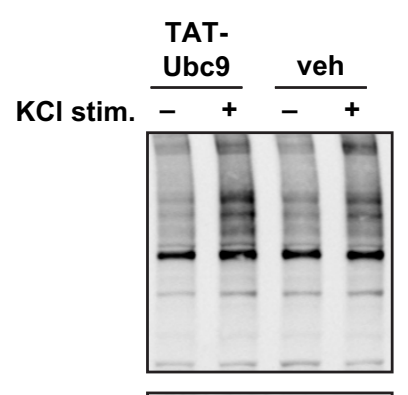

ß-actin
TAT-

Ubc9(DN) veh

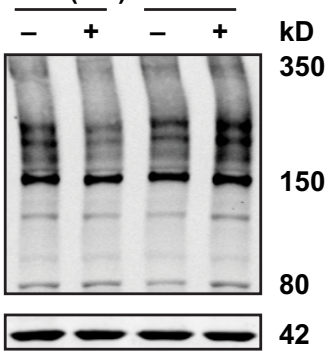

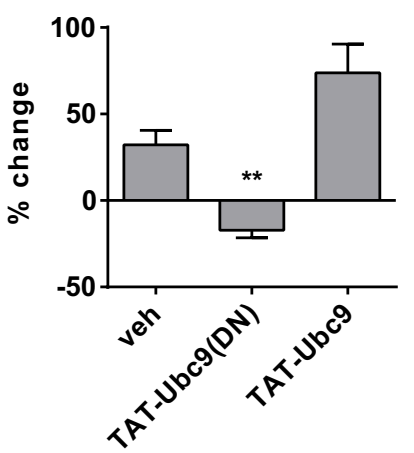

Figure $1 \mid$ Activity-dependent SUMOylation is blocked by dominant negative Ubc9 transduction. (a) Hippocampal slices were stimulated with high KCl and subsequently incubated for varying amounts of time in regular ACSF buffer. Representative western blots show high molecular weight SUMO2/3 conjugation. Quantification of blots indicated an increase in SUMOylation at the 5 minute post-depolarization time point. 0 min $\mathrm{n}=3$ each nonstimulated and stimulated, $5 \mathrm{~min} \mathrm{n}=3$ each, $30 \mathrm{~min} \mathrm{n}=3$ each. ANOVA $\mathrm{p}<0.001 ;{ }^{*} \mathrm{p}<0.05$ versus 0 min time point. (b) Schematic diagram of the recombinant TAT-Ubc9 expression construct. (c) In vitro SUMOylation assay with E2-25K substrate. Representative avidin-HRP western blot. Top band represents SUMOylated E2-25K substrate. Lane 1: positive control, lane 2: no ATP negative control, lane 3: reaction with TAT-Ubc9, lane 4: reaction with TAT-Ubc9(DN). (d) Representative Ubc9 western blot of acute hippocampal slices treated with vehicle or TAT-Ubc9 (1 hr $100 \mathrm{nM})$ and depolarized with high KCl. (e) Hippocampal slices were treated with vehicle (PBS), TAT-Ubc9 or TAT-Ubc9(DN) prior to stimulation by high potassium (5 minute post-stimulation incubation time). Representative western blots show high molecular weight SUMO2/3 conjugation. Vehicle $\mathrm{n}=11$ each non-stimulated and stimulated, TAT-Ubc9 $\mathrm{n}=3$ each, TAT-Ubc9 $(\mathrm{DN}) \mathrm{n}=7$ each. ANOVA $\mathrm{p}<0.0001 ;{ }^{*} \mathrm{p}<0.01$ versus all other groups. Data presented as means \pm SEM. See also Supplementary Figure S1.

or TAT-GFP controls (Fig. 2a, 2b). Baseline transmission was not altered during the TAT protein perfusions, and input-output curves were not affected by TAT-Ubc9 or TAT-Ubc9(DN) (Fig. 2c). In addition, we performed whole cell patch clamp experiments to investigate potential effects of SUMO inhibition on glutamate receptors known to underlie LTP. However, current-voltage curves for neither AMPA receptors nor NMDA receptors were altered by TAT-Ubc9(DN) (Fig. S2c, S2d). The effects of acute SUMOylation inhibition appear to be specific for long-term synaptic plasticity and do not impact basal transmission. 
a

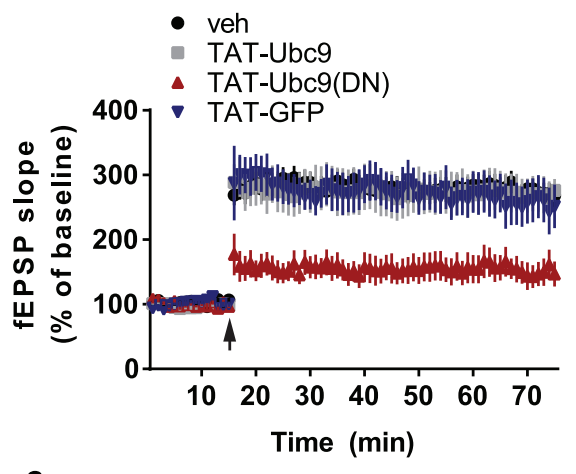

C

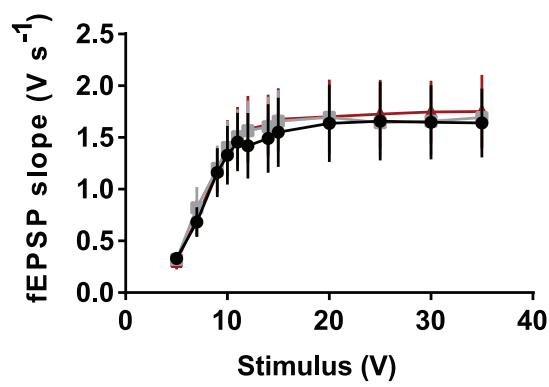

e

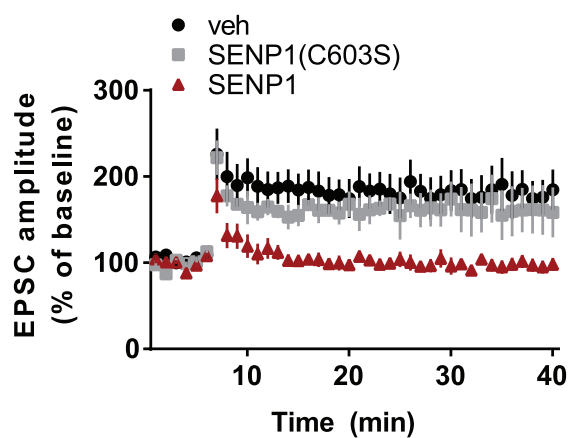

b

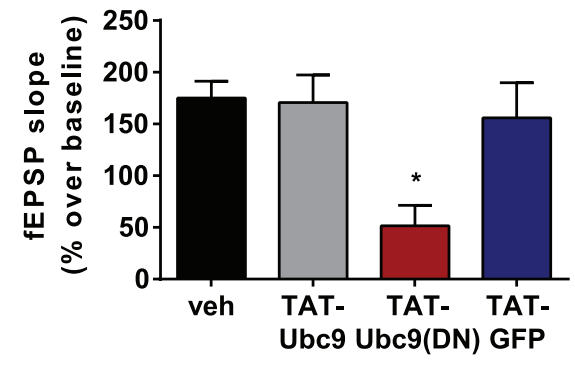

d

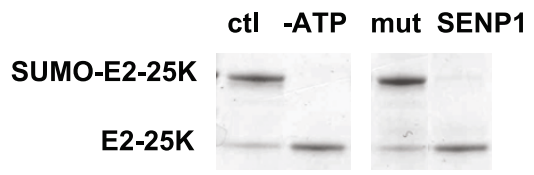

f

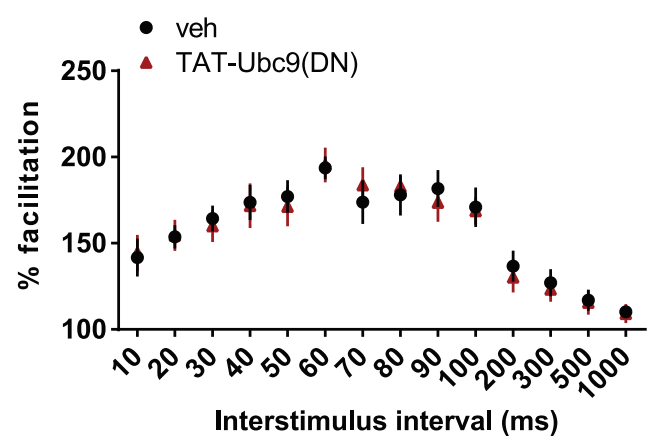

Figure $2 \mid$ SUMOylation is required for long-term potentiation. (a) LTP in area CA1 of hippocampal slices induced by theta burst stimulation of Schaffer collaterals. Treatments were perfused for 1 hour prior to LTP induction. Vehicle $n=14$, TAT-Ubc9 $n=9$, TAT-Ubc9(DN) $n=12$, TAT-GFP $n=7$. (b) Quantification of the last 10 minutes of LTP recordings. ANOVA of the averages, $\mathrm{p}=0.0004$; *TAT-Ubc9(DN) $\mathrm{p}<0.05$ versus all other groups. (c) Basal synaptic transmission, as measured by input-output curves, was not affected by TAT-Ubc9 or TAT-Ubc9(DN). (d) Recombinant GST-SENP1 has de-SUMOylating activity. In vitro SUMO2 conjugation assay using E2-25K substrate; representative avidin-HRP blots. The higher molecular weight band is SUMO2 conjugated substrate. Lane 1: positive control, lane 2: no ATP negative control, lane 3: reaction with GST-SENP1(C603S) inactive mutant, lane 4: reaction with GST-SENP1. (e) Whole cell patch clamp recordings of single-cell LTP in area CA1 induced by theta burst stimulation. Either vehicle, SENP1 or SENP1(C603S) inactive mutant was introduced intracellularly via the patch pipette. Vehicle $\mathrm{n}=7, \mathrm{SENP} 1(\mathrm{C} 603 \mathrm{~S}) \mathrm{n}=4, \mathrm{SENP} 1 \mathrm{n}=7$. ANOVA of the post-tetanic time points $\mathrm{p}<0.0001$. (f) Paired-pulse facilitation (PPF) in area CA1 of hippocampal slices. Vehicle and TAT-Ubc9(DN) $n$ $=6$ each. Data presented as means \pm SEM. See also Supplementary Figure S2.

We verified the effect of SUMO inhibition on long-term synaptic plasticity with another experimental approach. Instead of Ubc9 SUMO-conjugating enzyme, we used a SUMO-specific protease, SENP1, that has broad SUMO deconjugating activity ${ }^{32}$. Since SENP1 is a relatively large protein $(73 \mathrm{kD})$, it could not be utilized for the TAT transduction method. Instead, we directly administered either recombinant GST-tagged SENP1 or SENP1 catalytic subunit via patch pipette to the intracellular environment. In an in vitro assay, the recombinant SENP1 was found to have full de-
SUMOylating activity, while SENP1 with a previously-described inactivating mutation (C603S) did not (Fig. 2d) ${ }^{33}$. When included in the patch pipette, active SENP1, but not SENP1(C603S), fully blocked single-cell LTP in CA1 pyramidal neurons (Fig. 2e). Consistent with what we observed in the field potential experiments, baseline transmission was not affected (Fig. S2e).

While long-term synaptic plasticity in the form of LTP was significantly impaired by SUMO inhibition/deconjugation, we also investigated a form of short-term synaptic plasticity, paired-pulse 
a

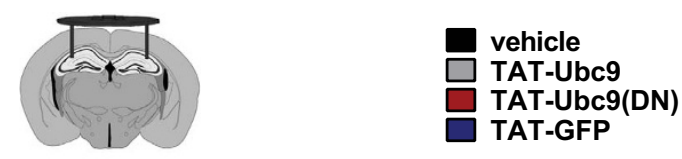

b

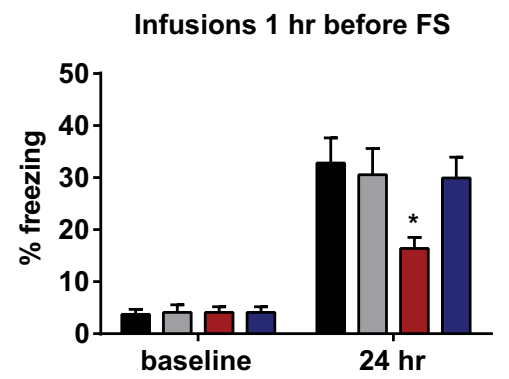

d

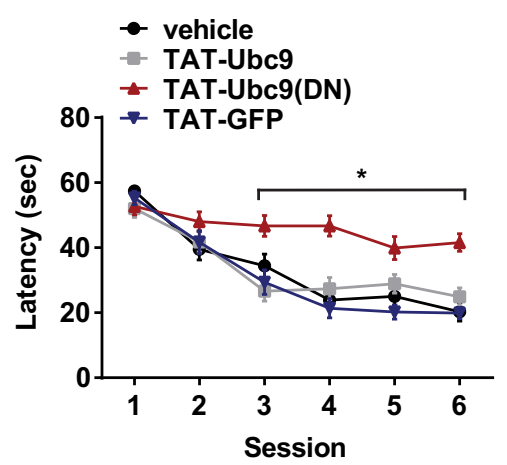

Infusions immediately after FS

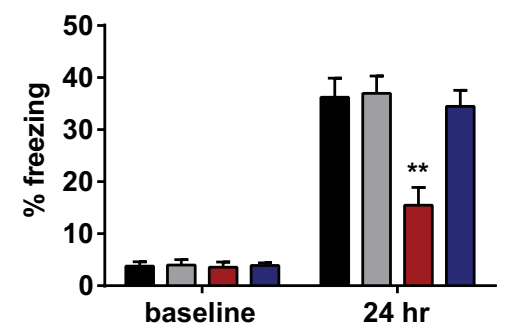

e

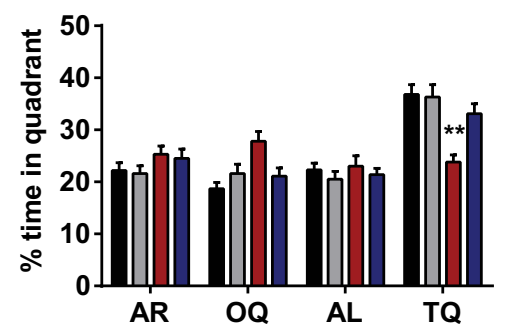

Figure 3 Inhibition of SUMO conjugation impairs hippocampal-dependent learning and memory. (a) Schematic diagram shows the placement of cannulas reaching the dorsal hippocampi. (b) Contextual fear conditioning behavioral task. Cannulated mice were infused 1 hour prior to foot shock training. Vehicle $\mathrm{n}=16$, TAT-Ubc9 $\mathrm{n}=8$, TAT-Ubc9(DN) $\mathrm{n}=16$, TAT-GFP $\mathrm{n}=12$. ANOVA of $\%$ freezing behavior 24 hours after foot shock training $\mathrm{p}=0.04 ;{ }^{*} \mathrm{p}<0.05$ versus all other groups. (c) Contextual fear conditioning behavioral task. Cannulated mice were infused immediately after foot shock training. Vehicle $n=10$, TAT-Ubc $9 n=12$, TAT-Ubc $9(\mathrm{DN}) \mathrm{n}=8$, TAT-GFP $\mathrm{n}=6$. ANOVA of $\%$ freezing behavior 24 hours after foot shock training $\mathrm{p}=0.0002 ; * \mathrm{p}<0.01$ versus all other groups. (d) Morris water maze behavioral task. Cannulated mice were infused after each training session. Vehicle $\mathrm{n}=11$, TAT-Ubc9 $\mathrm{n}=11$, TAT-Ubc9(DN) $\mathrm{n}=12$, TAT-GFP $\mathrm{n}=11$. ANOVA of time to reach the hidden platform $\mathrm{p}<0.0001$; ${ }^{*}$ TATUbc9(DN) group $\mathrm{p}<0.05$ versus all other groups starting from the third session. (e) Water maze probe trial. Mice infused with TAT-Ubc9(DN) spent significantly less time in the target quadrant (TQ). Adjacent right (AR), opposite quadrant (OQ), adjacent left (AL). ANOVA of time in target quadrant $\mathrm{p}$ $<0.0001$; **TAT-Ubc9(DN) group $\mathrm{p}<0.01$ versus all other groups. Data presented as means \pm SEM. See also Supplementary Figure S3.

facilitation (PPF), that is thought to be expressed presynaptically. TAT-Ubc9(DN) did not significantly affect PPF at any of the inter-stimulus intervals examined (Fig. 2f). Together with the postsynaptic LTP impairment observed in the SENP1 experiments, these data indicate that SUMOylation predominantly in the postsynaptic compartment is necessary for normal LTP.

SUMO inhibition impairs hippocampal-dependent learning and memory. Since SUMOylation is required for hippocampal LTP, we hypothesized that TAT-Ubc9(DN) would also impair learning and memory in vivo. In mice implanted with bilateral cannulas reaching the dorsal hippocampi (Fig. 3a), infusions of TAT-Ubc9 or TATGFP controls did not affect spatial learning and memory as tested in two hippocampal-dependent behavioral tasks: contextual fear conditioning (FC) and the Morris water maze (MWM) (Fig. 3).
However, infusions of TAT-Ubc9(DN) 20 minutes before foot shock training significantly impaired freezing behavior 24 hours later in the FC task (Fig. 3b). Administering TAT-Ubc9(DN) immediately after the foot shock training gave similar results (Fig. 3c), which indicate that the impairment effect is specific to memory consolidation ${ }^{34}$. In control experiments, there were no significant effects on performance in amygdala-dependent cued conditioning (Fig. S3a) or sensory thresholds (Fig. S3b).

In the Morris water maze task, which measures hippocampaldependent reference memory, infusions of TAT-Ubc9(DN) after each training session significantly impaired performance starting from the $3^{\text {rd }}$ session (Fig. 3d). In the probe trial, TAT-Ubc9(DN)treated mice spent significantly less time in the target quadrant compared to the other groups (Fig. 3e). Control experiments indicate that TAT transduction proteins did not affect either swimming speed or 

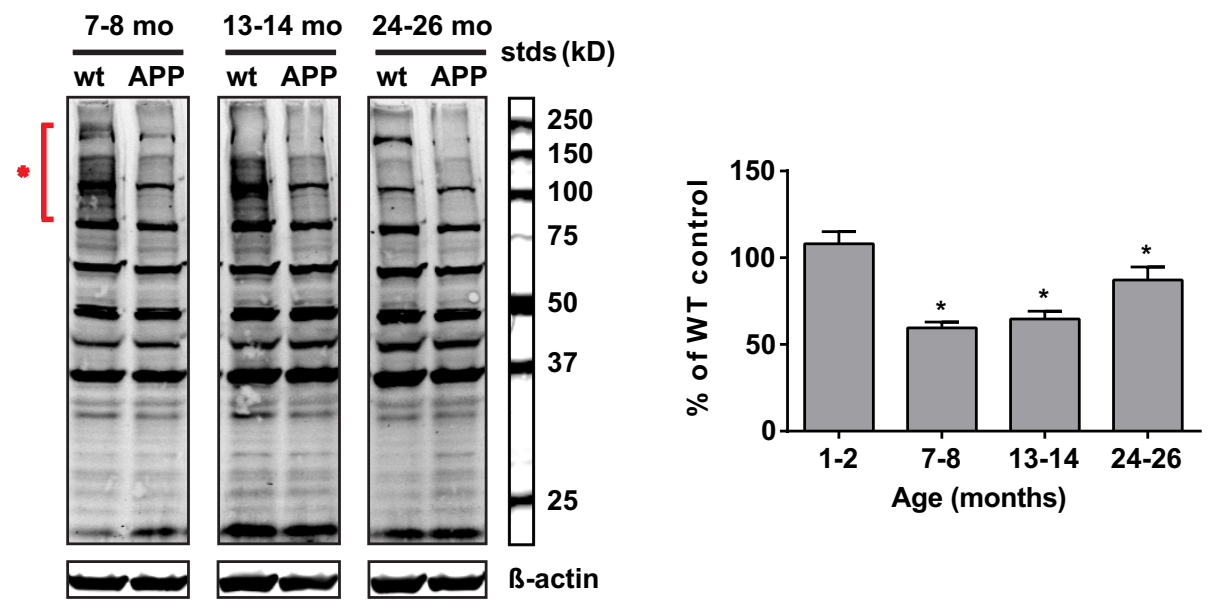

b
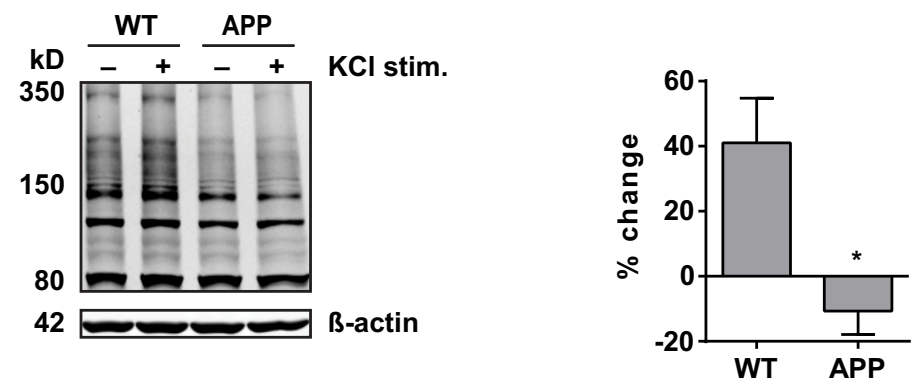

C
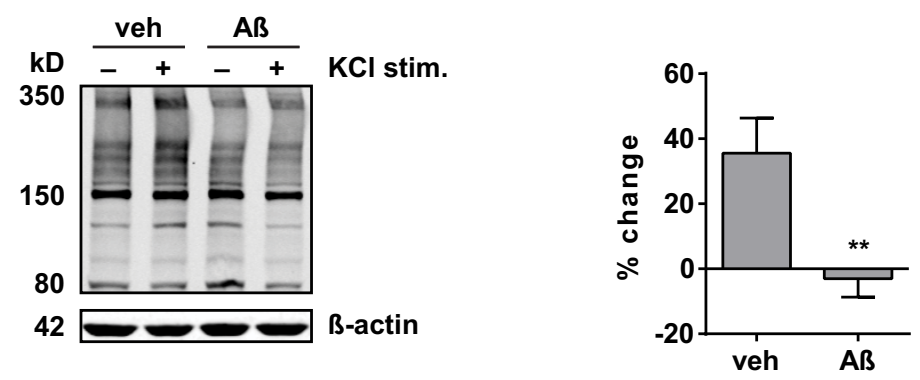

Figure 4 | SUMOylation is impaired by A $\beta$ and in Tg2576 APP-overexpressing mice. (a) SUMOylation is decreased in APP mice. Representative SUMO2/3 western blots with hippocampi from Tg2576 APP transgenic and wild-type littermate mice. 1-2 mo. $\mathrm{n}=6$ each WT and APP, 7-8 mo. $\mathrm{n}=3$ each, $13-14$ mo. $\mathrm{n}=4$ each; $24-26$ mo. $\mathrm{n}=3$ each. ANOVA $\mathrm{p}=0.0005 ;{ }^{*} \mathrm{p}<0.05$ vs. $1-2$ mo. group. Data presented as means \pm SEM, $\%$ of control group. (b) The depolarization-induced increase in SUMOylation at the 5 minute time point is not evident in APP mice compared to WT littermate controls. WT $n=6$, APP $n=5 ;{ }^{*} p=0.012$. (c) Exposure to $A \beta_{42}$ oligomers impaired the depolarization-induced SUMOylation at the 5 minute time point. Vehicle and $A \beta$-treated $n=6$ each; ${ }^{* *} \mathrm{p}=0.01$. Data presented as means $\pm \mathrm{SEM}, \%$ change in high molecular weight conjugation over nonstimulated control. See also Supplementary Figure S4.

time to reach a visible platform, which relate to motor coordination, vision and motivation (Fig. S3c, S3d). To rule out effects on exploratory behavior and anxiety, we also performed the open field test and found no differences between any of the groups, including the TATUbc9(DN)-treated mice (Fig. S3e, S3f). Overall, these data demonstrate the novel finding that SUMOylation is involved in and required for hippocampal-dependent learning and memory.

SUMOylation is dysregulated in APP-overexpressing transgenic mice. Alzheimer's disease, as a dementia characterized by synaptic and cognitive impairments, is known to involve dysregulation of post-translation modifications. In particular, pathological amyloid$\beta$ (A $\beta)$ conditions can potently disrupt signaling pathways involving
PTMs such as phosphorylation ${ }^{35-37}$. To investigate whether SUMOylation is altered by high $A \beta$ levels, we analyzed hippocampal tissue from Tg2576 transgenic mice, which overexpress the amyloid precursor protein (APP) and is a widely-used animal model of $\mathrm{AD}^{38}$. In aged APP mice (7-26 months old), we observed a significant decrease in SUMOylation compared to wild-type littermate controls (Fig. 4a). Consistent with our previous findings in $\mathrm{KCl}$ stimulated hippocampal slices, the decrease was most apparent in the high molecular weight SUMO2/3 conjugates $(>75 \mathrm{kD})$. The downregulation of SUMOylation primarily involves SUMO2/3 we detected minimal alteration of SUMO1 conjugates at any of the age points (Fig. S4a). In addition, we did not detect changes in the levels of the SUMO-conjugating enzyme, Ubc9 (Fig. S4b). 
The SUMO dysregulation was observed starting around 7-8 months of age; this is before the onset of widespread amyloid plaque deposition but does correlate with the presence of spatial memory deficits as well as detergent-insoluble $A \beta$ aggregates ${ }^{39}$. The decreased SUMOylation was apparent at all of the older ages examined, up to two years of age. At that point, the overall ratio of SUMOylation compared to wild-type controls appears to be increased from younger ages. This is due mostly to an apparent age-related decrease in SUMOylation in the wild-type mice (Fig. S4c). The decreased SUMOylation was again most evident in the high molecular weight area. These data indicate that SUMO dysregulation may also play a role in age-related cognitive decline.

Activity-induced SUMOylation is impaired by A $\beta$. To determine whether activity-induced SUMOylation is altered by $\mathrm{A} \beta$, we utilized acute hippocampal slices from 1 year old Tg2576 mice. Using the high-potassium stimulation protocol and 5 minute incubation time point, which induced the highest SUMOylation increase in our previous experiments, we found that the APP $\mathrm{Tg}$ slices were completely deficient in depolarization-dependent SUMOylation and lacked any significant SUMO changes upon stimulation (Fig. 4b). In contrast, slices from wild-type littermate controls were observed to have significant increases in high molecular weight SUMO conjugates, consistent with our previous data.

Although APP-overexpressing mice are useful models of amyloid pathology, there are also multiple other products of proteolytic APP processing besides $A \beta$ peptides. In addition, elevated $A \beta$ levels are chronically present in aged Tg2576 mice, which lead to a well-characterized multitude of pathological events in the brain. To pinpoint whether or not $A \beta$ is specifically and directly responsible for the impairment in SUMOylation, we also carried out these experiments with wild-type slices acutely exposed to A $\beta$ oligomers, which are thought to be the primary neurotoxins in $\mathrm{AD}$. A 20-minute incubation with $A \beta_{42}$ oligomers $(200 \mathrm{nM})$ prior to stimulation completely blocked the SUMOylation increase (Fig. 4c). In contrast to the Tg2576 mice, we did not consistently observe significant decreases in un-stimulated basal SUMOylation after $A \beta$ application (Fig. 4c, $5 a)$. Thus, while high $A \beta$, either acutely or chronically, can impair activity-dependent SUMOylation, longer-term A $\beta$ pathology is likely required for stable changes in basal SUMOylation. Overall, these experiments provide strong evidence that $A \beta$ peptides can impair activity-dependent SUMOylation in brain tissue.

Ubc9 transduction rescues $A \beta$-induced deficits in long-term potentiation. Given the $A \beta$-induced impairment effects on SUMOylation, we asked whether supplementing SUMO conjugation with wild-type TAT-Ubc9 could rescue activity-dependent SUMOylation. Using the high-potassium stimulation protocol, incubation of A $\beta$-treated hippocampal slices for 1 hour with TAT-Ubc9 $(100 \mathrm{nM})$ fully rescued the previously-observed SUMOylation impairment (Fig. 5a). Depolarization-induced SUMOylation was restored to normal levels comparable to the control groups. As previously noted, TAT-Ubc9 treatment alone did not significantly alter levels of basal non-stimulated SUMOylation (Fig. 5a). With stimulation, there was a trend towards increased activity-dependent conjugation, compared to vehicle control, although it did not reach significance. With this transduction system, it appears that the delivered TATUbc9 is supplemental and can be recruited under conditions of deficiency, such as that induced by $A \beta$. In addition, it should be noted that we did not detect changes in overall Ubc9 protein levels with acute $A \beta$ exposure (Fig. S5), which is consistent with our findings in the Tg2576 mice. The rescue of the SUMOylation impairment by TAT-Ubc9 suggests that other changes in Ubc9 function, such as localization or activity, or other SUMO regulatory factors may be involved in the deficits.

High concentration $A \beta$ exposure has been well-documented to potently inhibit synaptic function and plasticity. We therefore inves- tigated whether treatment with TAT-Ubc9 affects A $\beta$-induced deficits in long-term potentiation. Slices treated with $\mathrm{A} \beta_{42}$ oligomers (200 nM, $20 \mathrm{~min}$ ) exhibit significantly impaired LTP (Fig. 5b, 5c). However, perfusion with TAT-Ubc9 $(50 \mathrm{nM})$ for 1 hour prior to induction rescued LTP in slices also treated with $A \beta$ (Fig. 5b, 5c).

To investigate whether TAT-Ubc9 could also improve synaptic plasticity under chronic A $\beta$-related pathology, we perfused TATUbc9 onto hippocampal slices from 1 year-old Tg2576 mice. At this age, LTP was significantly impaired in the APP mice (Fig. 5d, 5e). Consistent with our acute A $\beta$ results, perfusion with TAT-Ubc9 $(50 \mathrm{nM})$ for 1 hour prior to LTP induction rescued the potentiation to wild-type levels (Fig. 5d, 5e). These experiments implicate dysregulated SUMOylation as a significant contributing factor underlying A $\beta$-induced synaptic dysfunction and LTP impairment.

Ubc9 transduction rescues $A \beta$-induced deficits in learning and memory. Since enhancing SUMOylation via Ubc9 transduction restored LTP, we asked whether A $\beta$-induced learning and memory deficits can also be rescued. In bilaterally cannulated mice, the infusion of $\mathrm{A} \beta_{42}$ oligomers $(200 \mathrm{nM})$ significantly impaired spatial learning and memory in both the contextual fear conditioning (FC) and the two-day radial arm water maze (RAWM) tasks (Fig. 6a, 6b). In the FC task, administration of $A \beta_{42} 15$ minutes prior to foot shock significantly reduced freezing behavior when tested 24 hours later (Fig. 6a). This A $\beta$-induced deficit was fully rescued by the infusion of TAT-Ubc9 immediately after the foot shock training. TAT-Ubc9treated mice demonstrated contextual memory similar to mice that did not receive $A \beta$.

In the RAWM task, $A \beta_{42}$ oligomers administered 15 minutes before the $1^{\text {st }}$ and $6^{\text {th }}$ training blocks significantly impaired performance in finding the hidden platform (Fig. 6b). Compared to the control group, the $A \beta$-treated mice failed to reach the learning criterion ( $\sim 1$ error) by the end of the second day. However, infusion of TAT-Ubc9 immediately after the $1^{\text {st }}$ and $6^{\text {th }}$ blocks restored performance in this task to normal levels comparable to the vehicle-treated group. In control experiments, TAT-Ubc9 infusions did not affect swimming speed or time to reach a visible platform (Fig. S6a, S6b).

Learning and memory impairments in APP transgenic mice can be rescued by Ubc9 transduction. We have demonstrated that supplementing SUMOylation can rescue learning and memory impairments caused by acute $A \beta$ exposure. However, we are particularly interested in the applicability of SUMO-modifying treatments for chronic A $\beta$-related pathology, as occurs in AD. Therefore, we also tested TAT-Ubc9 in 1 year-old Tg2576 APP mice, which have amyloid plaque pathology along with significant dendritic spine loss and synaptic dysfunction. Due to the more extensive pathology compared to the acute $A \beta$ model, we increased the dosing of TAT-Ubc9. In the FC task, TAT-Ubc9 was administered via cannulas 2 hours before, 20 minutes before and immediately after the foot shock training. When tested 24 hours later, the TAT-Ubc9-treated APP mice performed significantly better than vehicle-treated APP mice (Fig. 6c). In control experiments, TAT-Ubc9 did not affect amygdala-dependent cued conditioning or the sensory thresholds of the mice (Fig. S6c, S6d). These data demonstrate the spatial specificity of the TAT-Ubc9 hippocampal infusions. Cued conditioning by a tone conditioned stimulus is dependent on the amygdala and has been previously shown to be impaired in Tg2576 mice ${ }^{40}$; delivery of TAT-Ubc9 to the hippocampi via cannulas was not able to affect this type of learning.

A similar TAT-Ubc9 administration protocol was applied to the RAWM task. APP mice were infused with TAT-Ubc9 2 hours before, 20 minutes before and immediately after the $1^{\text {st }}$ and $6^{\text {th }}$ training blocks on day 1 and day 2, respectively. Towards the end of day 2, the TAT-Ubc9-treated APP mice performed significantly better at finding the hidden platform compared to vehicle-treated APP mice 

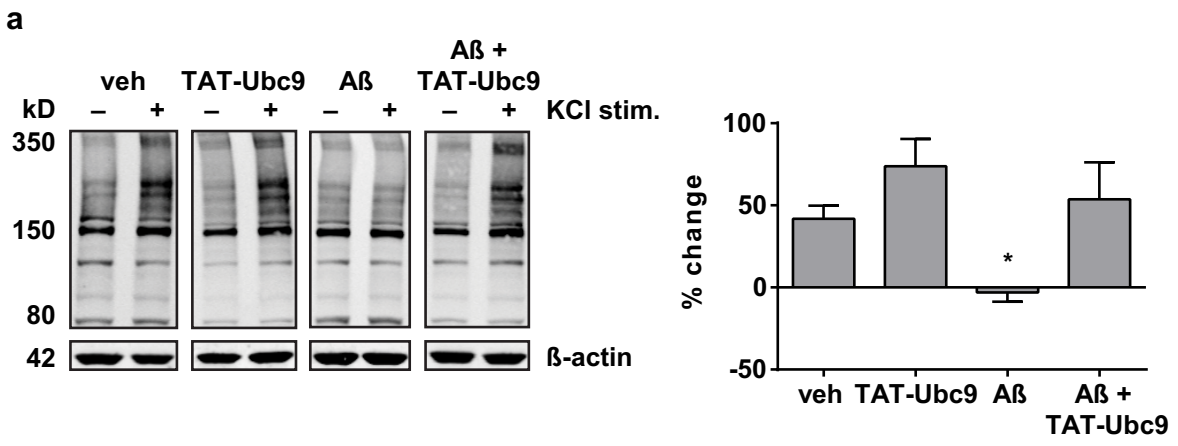

b

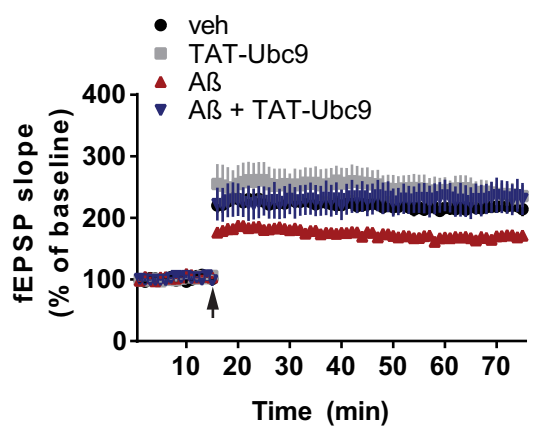

d

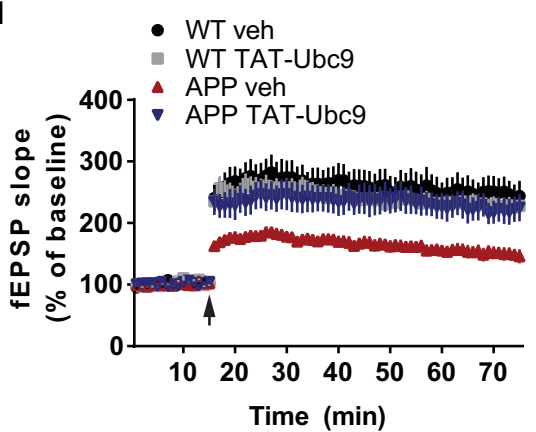

C

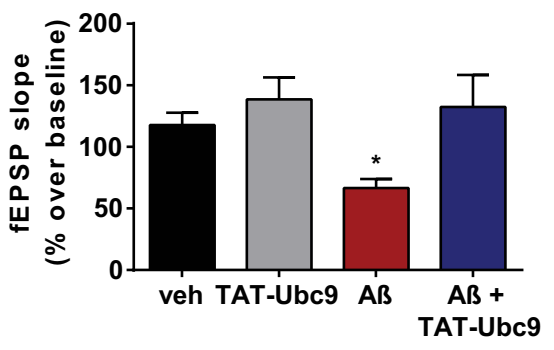

e

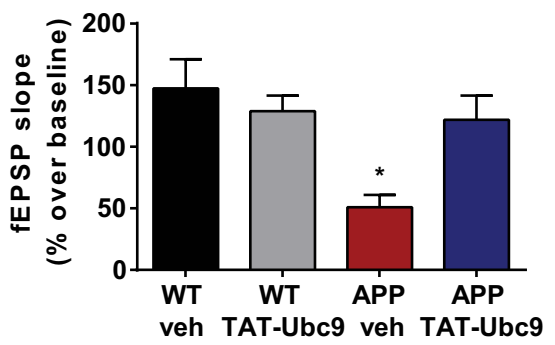

Figure $5 \mid$ Ubc9 transduction rescues A $\beta$-induced deficits in long-term potentiation. (a) Hippocampal slices were treated with vehicle (PBS), TATUbc9, $A \beta_{42}$ oligomers or A $\beta$ plus TAT-Ubc9 prior to stimulation by high potassium. Representative western blots show high molecular weight SUMO2/3 conjugation. Vehicle $\mathrm{n}=11$ each non-stimulated and stimulated, TAT-Ubc9 $\mathrm{n}=3$ each, A $\beta \mathrm{n}=6$ each, A $\beta$ plus TAT-Ubc9 $\mathrm{n}=6$ each. ANOVA $\mathrm{p}=$ $0.005{ }^{*} \mathrm{p}<0.05$ versus all other groups. (b) LTP in area CA1 of hippocampal slices induced by theta burst stimulation of Schaffer collaterals. Perfused treatments were prior to LTP induction and include: vehicle (PBS), TAT-Ubc9 (50 nM) for 1 hour, A $\beta_{42}$ oligomers $(200 \mathrm{nM})$ for $20 \mathrm{minutes}$, or combined A $\beta$ plus TAT-Ubc9. Vehicle $n=15$, TAT-Ubc9 $n=9, A \beta n=14, A \beta$ plus TAT-Ubc9 $n=9$. (c) Quantification of the last 10 minutes of LTP recordings. ANOVA of the averages of the last 10 minutes $\mathrm{p}=0.002 ;{ }^{*} \mathrm{p}<0.05$ versus all other groups. (d) LTP with hippocampal slices from Tg2576 mice. Perfused treatments were prior to LTP induction and include: vehicle (PBS) or TAT-Ubc9 (50 nM) for 1 hour. WT vehicle $\mathrm{n}=10$, WT TAT-Ubc9 $\mathrm{n}=13$, APP vehicle $\mathrm{n}=14$, APP TAT-Ubc9 $\mathrm{n}=15$. (e) Quantification of the last 10 minutes of LTP recordings. ANOVA of the averages of the last 10 minutes $\mathrm{p}=0.001 ;{ }^{*} \mathrm{p}<0.05$ versus all other groups. Data presented as means \pm SEM. See also Supplementary Figure S5.

(Fig. 6d). In control experiments, TAT-Ubc9 did not affect swimming speed or time to reach a visible platform (Fig. S6e, S6f). These experiments demonstrate that supplementation of SUMOylation can significantly improve cognitive function even with chronic A $\beta$ induced pathology.

An important question that arose was whether manipulating SUMOylation affected $A \beta$ levels. It is possible that the observed rescue effects in the $\operatorname{Tg} 2576$ mice were at least partially due to a change in $A \beta$ amounts. Previous studies in cell cultures indicate that SUMOylation can potentially regulate APP processing, although the conclusions in those studies were conflicting ${ }^{41-43}$. We investigated this possibility by performing ELISAs on APP hippocampal slices treated with TAT-Ubc9 (100 nM, 1 hour). In the soluble homogen- ization fractions, we found no significant effect of TAT-Ubc9 on either $A \beta_{40}$ or $A \beta_{42}$ levels (Fig. S6g). The rescue of synaptic plasticity and cognitive function in the $\mathrm{Tg} 2576$ mice by TAT-Ubc9 likely do not reflect significant changes in the soluble $A \beta$ pool, which constitutes the $A \beta$ fraction implicated in causing synaptic pathology in $\mathrm{AD}^{44}$.

SUMO is dysregulated in Alzheimer's disease hippocampus. We have shown that SUMO conjugation is significantly dysregulated by $\mathrm{A} \beta$ but can be rescued via Ubc 9 transduction. As a preliminary experiment on the potential translatability of our results to human Alzheimer's disease, we also analyzed post-mortem tissue samples from the hippocampal formation of six confirmed $\mathrm{AD}$ patients along 

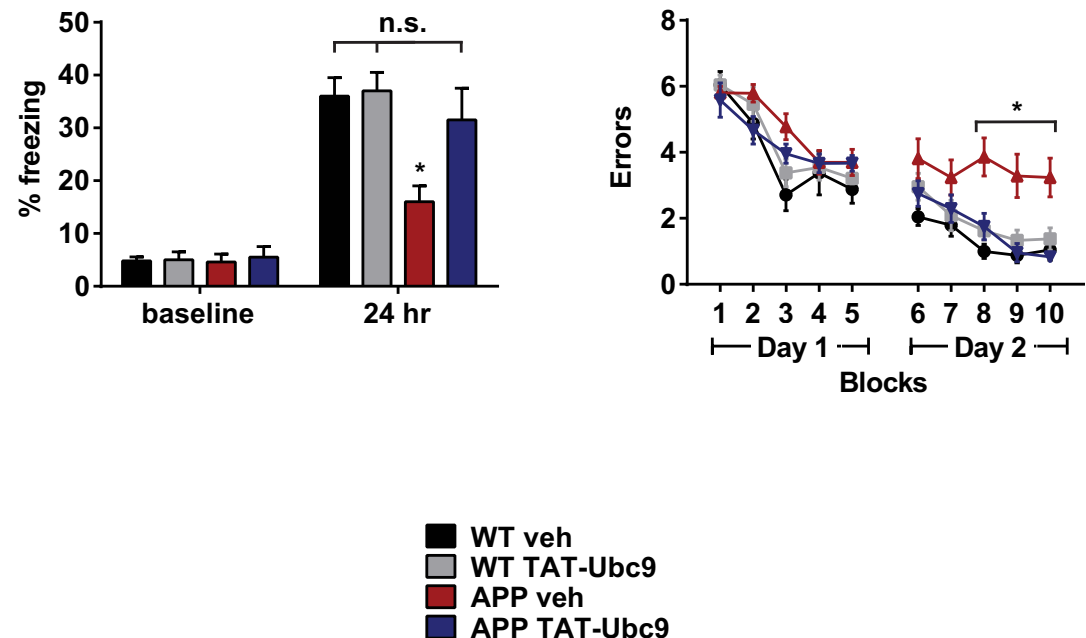

C

d
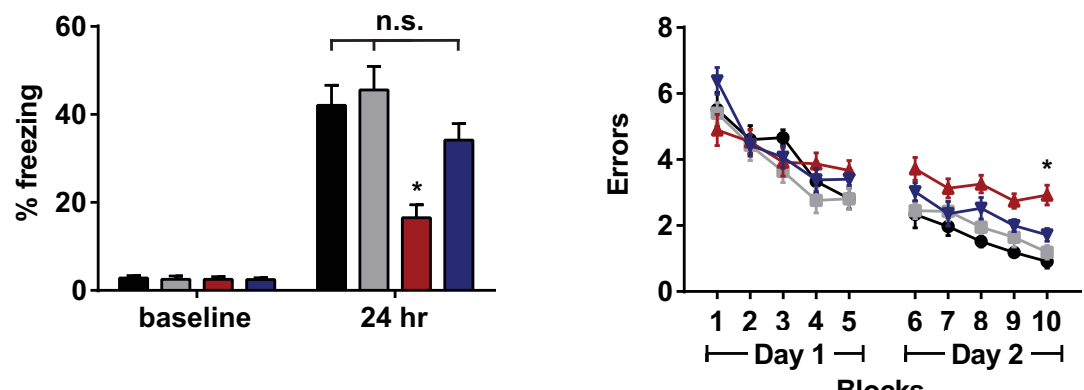

Figure 6 | Ubc9 transduction rescues hippocampal-dependent spatial learning and memory in AD mouse models. (a) Contextual fear conditioning behavioral task with acute $A \beta$ infusion model. Cannulated mice were each infused with 2 treatments: 1) 15 minutes prior to foot shock training and 2) immediately afterwards. Treatment groups include (infusion $1+$ infusion 2$)$ : vehicle $($ PBS $)+$ vehicle $(n=22)$, vehicle + TAT-Ubc9 $(n=13)$, A $\beta_{42}$ oligomers + vehicle $(n=14) ; A \beta_{42}$ oligomers + TAT-Ubc9 $(n=13)$. ANOVA of $\%$ freezing behavior 24 hours after foot shock training $\mathrm{p}=0.002$; ${ }^{*} \mathrm{p}<0.05$ versus all other groups. (b) Radial arm water maze behavioral task with acute $A \beta$ infusion model. Cannulated mice were infused for training sessions 1 and 6 with 2 treatments: 1) 15 minutes prior and 2) immediately after the session. Treatment groups include (infusion $1+$ infusion 2 ): vehicle $($ PBS $)+$ vehicle $(n=8)$, vehicle + TAT-Ubc9 $(n=8), A \beta_{42}$ oligomers + vehicle $(n=7) ; A \beta_{42}$ oligomers + TAT-Ubc9 $(n=8)$. ANOVA of number of errors $\mathrm{p}=0.0002 ;{ }^{*} \mathrm{~A} \beta+$ vehicle group $\mathrm{p}<0.05$ versus all other groups. (c) Contextual fear conditioning behavioral task with Tg2576 mice. Cannulated APP and wild-type (WT) mice were infused with 3 treatments: 1) 2 hours prior to foot shock training, 2) 20 minutes prior and 3) immediately afterwards. WT vehicle (PBS) $\mathrm{n}=11$, WT TAT-Ubc9 $\mathrm{n}=13$, APP vehicle $\mathrm{n}=13$; APP TAT-Ubc9 $\mathrm{n}=13$. ANOVA of $\%$ freezing behavior 24 hours after foot shock training $\mathrm{p}<0.0001{ }^{*} \mathrm{p}<0.05$ versus all other groups. (d) Radial arm water maze behavioral task with Tg2576 mice. Cannulated APP and WT mice were infused for training sessions 1 and 6 with 3 treatments: 1) 2 hours prior to the session, 2) 20 minutes prior and 3) immediately after the session. WT vehicle (PBS) $\mathrm{n}=11$, WT TAT-Ubc9 $\mathrm{n}=14$, APP vehicle $\mathrm{n}=13$; APP TAT-Ubc9 $\mathrm{n}=14$. ANOVA of number of errors $\mathrm{p}<0.0001$; *APP vehicle group $\mathrm{p}<0.05$ versus all other groups. Data presented as means \pm SEM; n.s. $=$ not significant. See also Supplementary Figure S6.

with six non-demented controls (Table S1). Similar to what we observed in the aged Tg2576 mice, the AD samples exhibited significantly less high molecular weight SUMO2/3 conjugates compared to controls (Fig. 7). This decrease was the most consistent in the high molecular weight region $(>75 \mathrm{kD})$, where the AD samples had about half of the SUMOylation levels seen in non-demented controls. This dysregulation is again specific for SUMO2/3, as levels of SUMO1 conjugates were not significantly altered in the AD samples (Fig. S7a). In addition, similar to the APP-overexpressing mice, the decreased SUMOylation is not due to an overall change in Ubc9 protein levels (Fig. S7b); changes in other aspects of Ubc9 function and/or localization or changes in other SUMO enzymes and regulatory factors cannot be ruled out and remain to be assessed in future studies. Overall, our data with the human brain samples implicate dysregulated SUMOylation as a novel factor in $\mathrm{AD}$ pathology and are fully consistent with our findings in the mouse model.

\section{Discussion}

SUMOylation in synaptic plasticity and cognition. In this study, we have demonstrated for the first time that SUMOylation is required for long-term potentiation and hippocampal-dependent cognition. 

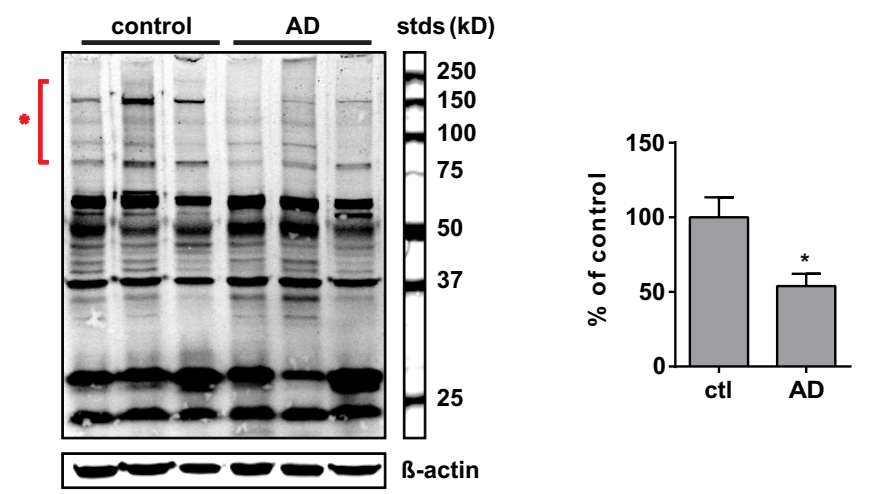

Figure 7 SUMOylation is dysregulated in human Alzheimer's disease hippocampal tissue. SUMOylation is decreased in AD. Representative SUMO2/3 western blot with post-mortem hippocampal formation tissue from $\mathrm{AD}$ and control patients. Starred bracket indicates the analyzed high molecular weight region exhibiting the most significant reduction of SUMO conjugates. Control and AD $n=6$ each; ${ }^{*} \mathrm{p}=0.015$. Data presented as means \pm SEM, \% of control group. See also Supplementary Figure S7, Table S1.

Our results are consistent with recent studies showing that SUMOylation can be modulated by either direct depolarization or a glycine-induced "ChemLTP" protocol in dispersed neuronal cultures $^{45,46}$. The results from those studies conflict with each other although both do indicate that SUMOylation levels are regulated within a narrow post-stimulus time frame ( 5 minutes), similar to our data presented here. While one study found that SUMO2/3, but not SUMO1, conjugation levels are decreased following depolarization $^{45}$, the other study found that there is an increase in only SUMO1 after ChemLTP ${ }^{46}$. Such discrepancies are likely due to the differing stimulation protocols used with embryonically-derived culture systems, which generally do not reflect the activity of adult brain tissue. We have now shown that dynamic SUMO2/3 regulation does occur in acute hippocampal slices that are stimulated with either direct depolarization or electrical (theta burst) induction of LTP.

In contrast to SUMO2/3, we observed minimal global effects involving the SUMO1 paralog. Previous studies indicate that there is paralog-specific functionality, with distinct roles and differing target subsets ${ }^{20,47,48}$. For example, in mammalian cells, the pool of unconjugated SUMO2/3 is generally much larger than that of $\mathrm{SUMO}^{20}$. The N-terminal of SUMO2/3 also contains the SUMO consensus site, which allows for the formation of poly-SUMO chains ${ }^{23}$. In terms of molecular dynamics, SUMO1 conjugation has been found to be much slower compared to SUMO2/3 ${ }^{49}$. Further supporting this observation, SUMO1 deconjugation is mediated mostly by SENP1 and, with lower efficiency, SENP2; in contrast, all six of the known human SENP proteases have activity for SUMO2/3 32,50 . Thus, it appears that SUMOylation by SUMO2/3 has a potentially larger dynamic capacity compared to SUMO1, which may make SUMO2/3 particularly well-suited for rapidly regulated processes such as synaptic plasticity. The high molecular weight region of SUMO2/3 conjugates, where we observed the most significant global changes following neuronal stimulation, likely includes multiple poly-SUMOylated substrates that are particularly labile to changes in SUMO regulation. Although we do not rule out significant involvement and contribution of SUMO1 to synaptic plasticity, SUMO2/3 appears to be better-suited for the purposes of assaying and analyzing large-scale, dynamic changes in SUMOylation. In this study, we focus on SUMO2/3 for assaying purposes but we do not exclude the possibility that the effects of TAT-Ubc9 transduction may be partially mediated by SUMO1 modifications.

Along with other PTMs such as phosphorylation, SUMOylation likely plays widespread roles in the signaling networks that regulate synaptic plasticity. How analogous SUMOylation is to these other PTMs remains to be fully determined. However, it is now known that SUMOylation, similar to phosphorylation, is capable of regulating multiple aspects of substrate function including protein localization and intermolecular interactions ${ }^{10}$. SUMO has the potential to participate broadly in synaptic signaling networks and as such, likely involves numerous substrate targets on a relatively large scale. Using whole-tissue analyses to look for these broad changes in regulation, we have now demonstrated that SUMOylation is indeed dynamically regulated by neuronal activation and is necessary for normal longterm potentiation.

Comparing further with phosphorylation, prominent early studies in the learning and memory field observed increases in kinase activity and overall protein phosphorylation following various types of stimulation ${ }^{51-57}$. In addition, analogous to our findings with TATUbc9(DN), inhibiting phosphorylation was shown to impair LTP as well as learning and memory ${ }^{58-62}$. However, as evidenced by subsequent studies on specific phosphorylated substrates, it is important to note that the actual phosphorylation states of specific proteins are regulated by networks of kinases and phosphatases and could be either modified or de-modified upon plasticity induction.

We hypothesize that the involvement of SUMOylation in synaptic plasticity is similar. In this study, we have discovered that SUMOylation levels are dynamically regulated by neuronal activation and LTP-inducing stimulation. Furthermore, such regulation is necessary for learning and memory, since inhibiting SUMOylation with TAT-Ubc9(DN) causes synaptic and cognitive impairments. Although we observed overall increases in the high molecular weight conjugates upon stimulation, indicating broad changes in SUMO regulation, the SUMOylation states of specific proteins are individually regulated and may not necessarily be modified in the same direction or time frame. In our experiments, we focused on overall high molecular weight SUMO conjugation, which serves as an assay for detecting global changes in SUMO regulation. Future research will elucidate the specific protein targets that undergo regulated SUMOylation (or de-SUMOylation) as part of the mechanisms underlying long-term potentiation and memory.

There are several potential candidates which have been recently described to be SUMO targets in neurons. For example, the GluK2 kainate receptor subunit is SUMOylated upon stimulation ${ }^{16}$, and Arc, a well-studied immediate early gene, was recently found to be SUMOylated as part of a homeostatic synaptic scaling mechanism ${ }^{26}$. Furthermore, GluK2 SUMOylation was recently discovered to be required for another form of synaptic plasticity, long-term depression, at mossy fiber synapses ${ }^{63}$. The SUMOylation of neuronal substrates such as these could potentially be involved in the SUMO-regulated effects on hippocampal-dependent cognition that we have demonstrated and remain to be investigated in follow-up studies. Our current study opens new avenues of research into SUMO as a novel PTM regulator of long-term potentiation and cognition.

SUMOylation in Alzheimer's disease. In this study, we have also discovered that activity-dependent SUMOylation is impaired by high A $\beta$ levels and can be rescued by supplementing SUMO conjugation capacity with TAT-Ubc9. Since we observed that SUMO inhibition on its own is sufficient to cause learning and memory deficits, the SUMOylation impairments by A $\beta$ likely contribute to the synaptic and cognitive dysfunction of AD. There is growing evidence that the normal regulation of SUMO can indeed become disrupted under neuropathological conditions and further contribute to disease pathways ${ }^{64}$. In particular, previous studies have implicated SUMOylation in neurodegenerative diseases such as Huntington's disease and spinocerebellar ataxias ${ }^{15,65}$.

In the $\mathrm{AD}$ field, biochemical and genetic studies have pointed to possible links with SUMOylation. A previous study that also utilized the Tg2576 APP-overexpressing mouse model found that cortical, 
but not hippocampal, high molecular weight SUMO2/3 conjugates are significantly decreased in transgenic mice ${ }^{66}$. SUMO1 conjugate levels were not observed to be significantly altered. A more recent study with the Tg2576 mouse model confirmed that SUMO2 conjugation levels are decreased in an age-dependent manner in cortical and hippocampal tissue, although SUMO1 conjugate levels were observed to be increased in this study ${ }^{67}$. The general finding that SUMO2/3 conjugation is decreased in $\operatorname{Tg} 2576$ mice is consistent with our data presented here; however, the discrepancies in SUMO1 data, affected brain regions and extent of SUMOylation changes are likely due to differences in experimental and analytical methods and remain to be investigated in follow-up studies.

Single nucleotide polymorphisms (SNPs) associated with AD have been discovered in genes encoding SUMO enzymes, including Ubc9 and a homolog of the SUMO activating enzyme 2 (SAE2) ${ }^{68-70}$. The Ubc9 SNPs were discovered in a population of patients with either sporadic late-onset $\mathrm{AD}$ or mild cognitive impairment $(\mathrm{MCI})^{68}$. A SNP in the Ubc9 gene (UBE2I) was found to be significantly associated with sporadic AD.

In our study, we did not find significant changes in Ubc9 protein levels in the hippocampi of $\mathrm{AD}$ patients or APP-overexpressing mice. This is consistent with a previous study that also did not detect changes in Ubc9 levels in the transgenic mice ${ }^{66}$. However, it is possible that alterations in other aspects of Ubc9 regulation may affect its function under $A \beta$-induced pathology. This is supported by our experiments demonstrating the rescue of synaptic and cognitive function with the transduction of functional TAT-Ubc9 enzymes. For example, Ubc9 is normally localized in neuronal processes ${ }^{16}$. With AD pathology, which includes disruption of protein trafficking, Ubc9 localization may become abnormal and lead to SUMOylation deficits in parts of the cell, such as the synaptic compartments; any such Ubc9 deficiencies would be amendable to rescue by the administration of TAT-Ubc9 enzymes. Alternatively, Ubc9 enzymatic activity could also become impaired or dysregulated. Recently, it was discovered that Ubc9 SUMO conjugation activity can be regulated by CDK1-dependent phosphorylation ${ }^{71}$. Dysregulation of the cyclin dependent kinases (CDKs), including CDK1, has been previously associated with $\mathrm{AD}^{72,73}$; thus, altered Ubc9 phosphorylation may occur with $\mathrm{AD}$ and lead to impaired or dysregulated SUMO conjugation. It is important to note that in addition to Ubc9, there may also be changes in the other enzymes and regulatory factors of the SUMO system, including E3 ligases and deSUMOylating proteases. These are open questions that remain to be addressed in future investigations. As a starting point, our current study has demonstrated that there is impaired SUMOylation with $\mathrm{AD}$ that is caused, at least in part, by pathological $\mathrm{A} \beta$ oligomers.

While we have discovered that SUMOylation is altered downstream of $A \beta$ with $A D$ pathology, there is also evidence for upstream effects on APP processing ${ }^{41-43}$. However, the data on whether or not APP is directly SUMOylated and whether SUMOylation impacts A $\beta$ levels are currently conflicting and unclear. We investigated this possibility and found that altering SUMOylation with TAT-Ubc9 did not significantly affect soluble $A \beta$ levels in hippocampal tissue from APP-overexpressing mice. However, altering SUMOylation may impact other aspects of APP processing, including levels of other cleavage products and its regulation by neuronal activity; these other factors currently cannot be ruled out and may play a role in the rescue effects we observed. Ultimately, SUMOylation may be involved in both downstream and upstream pathways in $\mathrm{AD}$ pathogenesis. Besides APP, the microtubule associated protein tau - another major protein culprit implicated in multiple neurodegenerative diseases has also been described as a SUMO substrate with cross-talk PTM interactions with phosphorylation and ubiquitination ${ }^{74}$. With both downstream and upstream involvement in $\mathrm{AD}$, there could be a pathological feedback signaling in the SUMO system - abnormal $\mathrm{A} \beta$ aggregates impair SUMOylation, as we have demonstrated in this study, and impaired SUMOylation could further contribute to $\mathrm{AD}$ pathogenesis through modulation of $\mathrm{A} \beta$ and tau proteins. Combined with the downstream effects on synaptic signaling pathways, perturbations of $A \beta$ and tau would feed back to further dysregulate SUMOylation in a pathogenic cycle.

We have demonstrated that supplementing SUMO conjugation capacity with TAT-Ubc9 is sufficient for countering A $\beta$-induced synaptic and cognitive deficits, which suggests that SUMO-targeted compounds may be relevant for $\mathrm{AD}$ therapeutics. Especially striking is our finding that even chronic $A \beta$-induced synaptic and cognitive dysfunction in aged APP-overexpressing mice can be rescued by altering SUMOylation. Acutely transducing Ubc9 in hippocampal slices rescues LTP, and same-day infusions of TAT-Ubc9 significantly improves performance in spatial learning and memory tasks. These results strongly suggest that dysregulated SUMO plays an important role in the cognitive impairments caused by $A \beta$. Furthermore, the SUMO dysregulation appears to be amendable to modification even with relatively established neuropathology; besides elevated A $\beta$ levels and plaques, one year old Tg2576 mice also exhibit significant decreases in spine densities ${ }^{75-77}$. The rapid improvements in learning and memory upon Ubc9 transduction are striking and suggest that restoring SUMOylation, likely of downstream signaling proteins, can rescue and compensate for the loss of synapses at this stage of the disease. Currently, SUMOylation-directed approaches are most active in the cancer field. Given the role of SUMO in genomic maintenance, targeting Ubc9 and overall SUMOylation is not a likely approach for AD therapeutic strategies. However, with the continuing discovery of SUMOylated neuronal proteins and SUMO regulatory factors, specific ones involved in synaptic signaling could eventually be targeted for the treatment of neurodegeneration-related cognitive decline. Our findings suggest that this approach could have a significant effect even with established pathology.

Our study is the first to demonstrate the critical role of SUMOylation in learning and memory along with the impairment of such functions with Alzheimer's disease pathology. Given the growing evidence for the involvement of SUMO in neuron-specific processes, we believe SUMOylation will emerge as a key regulator of both normal synaptic functioning and neuropathogenesis.

\section{Methods}

Animals. $\mathrm{Tg} 2576^{38}$ or C57BL/6J mouse colonies were housed at Columbia University and maintained on a 12-hour light/dark cycle. Unless otherwise specified, 12-14 month old Tg2576 males were used. For experiments with only wild-type mice, 4-6 month old males were used (3-4 week old for whole-cell patch clamp experiments). Mice were sacrificed by cervical dislocation followed by decapitation. For biochemical studies, hippocampi were snap frozen in liquid nitrogen. All protocols involving animals were approved by Columbia University and the Institutional Animal Care and Use Committee (IACUC); experiments involving animals were performed in accordance with the relevant approved guidelines and regulations.

Human brain tissue. Frozen post-mortem hippocampal tissues were obtained from the New York Brain Bank at Columbia University Medical Center, as approved by the Institutional Review Board (IRB). All samples were originally collected in accordance with the relevant approved guidelines and regulations, including those regarding subject consent and sample de-identification. Alzheimer's disease samples were obtained from patients with autopsy-confirmed AD (see Table S1). Controls did not have any major neuropathology and did not meet National Institutes of HealthReagan Institute criteria for AD.

Amyloid-beta peptide preparation. $\mathrm{A} \beta_{42}$ peptides (American Peptide Company) were prepared as previously described ${ }^{78,79}$. Briefly, lyophilized peptides were dissolved to $1 \mathrm{mM}$ in hexafluoroisopropanol and dried to produce peptide films. Prior to use, films were dissolved in anhydrous dimethylsulfoxide to $5 \mathrm{mM}$ and bath sonicated. To prepare oligomers, the DMSO solution was diluted to $100 \mu \mathrm{M}$ in PBS and incubated overnight at $4{ }^{\circ} \mathrm{C}$. The quality of the $\mathrm{A} \beta$ preparations were routinely controlled using immunoblot analyses.

Purification of recombinant proteins. TAT-Ubc9 and TAT-Ubc9(DN) constructs were made by subcloning murine Ubc9 or Ubc9(C93S) (gifts from Dr. Olli Jänne, U. of Helsinki, Finland) into a TAT-HA-6xHis vector (gift from Dr. Steven Dowdy, U. of California, San Diego). The TAT-GFP construct was made by sub-cloning GFP from 
the pEGFP-1 plasmid (Clontech) into the TAT vector. GST-SENP1 and GSTSENP1(C603S) constructs were gifts from Dr. Steve Goldstein, U. of Chicago. Protein expression in E. coli and purification via the $6 \mathrm{xHis}$ tag/GST tag were performed according to standard procedures (see Extended Experimental Procedures).

SUMO conjugation assay. In vitro SUMO1 and SUMO2 conjugation assays (Boston Biochem) were performed according to the manufacturer's protocols. Reaction components included biotinylated E2-25K SUMOylation substrate, E1 conjugating enzyme (SAE1/SAE2), SUMO, Mg-ATP and Ubc9 (or TAT-Ubc9). Assays were processed with avidin-HRP western blotting.

\section{Electrophysiology and high-potassium stimulation. Following decapitation,} hippocampi were rapidly removed and transverse slices $(400 \mu \mathrm{m})$ were cut. Slices were allowed to recover for at least 90 minutes prior to recording. Extracellular field potential and whole cell patch clamp recordings were performed with the CA3-CA1 pathway according to standard procedures (see Extended Experimental Procedures). LTP was induced using theta-burst stimulation. All experiments were performed with interleaved controls. For high-potassium stimulation studies, the depolarization buffer included $50 \mathrm{mM} \mathrm{KCl}$ in regular ACSF, with decreased $\mathrm{NaCl}$ concentration $(74 \mathrm{mM})$ to maintain osmolarity. A stimulation time of 2 minutes was used for all high- $\mathrm{KCl}$ experiments.

Immunoblotting. Tissues were homogenized in RIPA buffer containing protease and phosphatase inhibitors, as well as $20 \mathrm{mM} \mathrm{N}$-ethylmaleimide to prevent SUMO deconjugation. Western blot analyses of the soluble fractions were carried out according to standard procedures (see Extended Experimental Procedures). The antibodies used include: SUMO2/3 (Invitrogen), SUMO1 (Cell Signaling), Ubc9 (BD), $\beta$-actin (Abcam), TUJ1 (Promega), HA tag (Covance). Blot images were analyzed using Image (NIH) software. Data were normalized to a loading control ( $\beta$ actin).

Cannula implantation and behavioral studies. Cannulas were implanted as previously described ${ }^{79}$. Briefly, mice were anesthetized, and bilateral cannulas were implanted to reach the dorsal hippocampi. The mice were allowed to recover for 6-8 days following implantation. Infusions were done with $1 \mu \mathrm{L}$ of vehicle/treatment over 1 minute (per side). For FC studies, $A \beta$ infusions were performed 15 minutes prior to the foot shock training. For RAWM studies, $A \beta$ infusions were performed 15 minutes prior to the $1^{\text {st }}$ and $6^{\text {th }}$ sessions. TAT-Ubc9 infusions were performed at time points described in the text. The behavioral tasks were performed as previously described (see Extended Experimental Procedures).

A $\beta$ ELISAs. $A \beta_{(x-40)}$ and $A \beta_{(x-42)}$ ELISAs (Covance) were performed according to manufacturer's protocols. Tissues were homogenized in tris-buffered saline containing protease inhibitors, and the soluble fractions were immediately used for the ELISAs.

Statistical analyses. All data are presented as means \pm SEM. Data were analyzed by two-tailed Student's t-test or ANOVA plus post-hoc Tukey's multiple comparisons test using Prism (GraphPad) software. The threshold for significance was set at $\mathrm{p}<$ 0.05 in all analyses.

1. Soderling, T. R. \& Derkach, V. A. Postsynaptic protein phosphorylation and LTP. Trends Neurosci. 23, 75-80 (2000).

2. DiAntonio, A. \& Hicke, L. Ubiquitin-dependent regulation of the synapse. Annu. Rev. Neurosci. 27, 223-246 (2004).

3. Fukata, Y. \& Fukata, M. Protein palmitoylation in neuronal development and synaptic plasticity. Nat. Rev. Neurosci. 11, 161-175 (2010).

4. Routtenberg, A. \& Rekart, J. L. Post-translational protein modification as the substrate for long-lasting memory. Trends Neurosci. 28, 12-19 (2005).

5. Lee, V. M., Goedert, M. \& Trojanowski, J. Q. Neurodegenerative tauopathies. Annu. Rev. Neurosci. 24, 1121-1159 (2001).

6. Ross, C. A. \& Poirier, M. A. Protein aggregation and neurodegenerative disease. Nat. Med. 10 Suppl, S10-17 (2004).

7. Snyder, E. M. et al. Regulation of NMDA receptor trafficking by amyloid-beta. Nat. Neurosci. 8, 1051-1058 (2005).

8. Hsieh, H. et al. AMPAR removal underlies Abeta-induced synaptic depression and dendritic spine loss. Neuron 52, 831-843 (2006).

9. Martin, S., Wilkinson, K. A., Nishimune, A. \& Henley, J. M. Emerging extranuclear roles of protein SUMOylation in neuronal function and dysfunction. Nat. Rev. Neurosci. 8, 948-959 (2007).

10. Geiss-Friedlander, R. \& Melchior, F. Concepts in sumoylation: a decade on. Nat. Rev. Mol. Cell Biol. 8, 947-956 (2007).

11. Johnson, E. S. Protein modification by SUMO. Annu. Rev. Biochem. 73, 355-382 (2004).

12. Zhang, F. P. et al. Sumo-1 function is dispensable in normal mouse development. Mol. Cell. Biol. 28, 5381-5390 (2008).

13. Lee, Y. J. et al. Elevated Global SUMOylation in Ubc9 Transgenic Mice Protects Their Brains against Focal Cerebral Ischemic Damage. PloS one 6, e25852 (2011).

14. Watanabe, M., Takahashi, K., Tomizawa, K., Mizusawa, H. \& Takahashi, H. Developmental regulation of Ubc9 in the rat nervous system. Acta Biochim. Pol. 55, 681-686 (2008).
15. Wilkinson, K. A., Nakamura, Y. \& Henley, J. M. Targets and consequences of protein SUMOylation in neurons. Brain Res. Rev. 64, 195-212 (2010).

16. Martin, S., Nishimune, A., Mellor, J. R. \& Henley, J. M. SUMOylation regulates kainate-receptor-mediated synaptic transmission. Nature 447, 321-325 (2007).

17. Feligioni, M., Nishimune, A. \& Henley, J. M. Protein SUMOylation modulates calcium influx and glutamate release from presynaptic terminals. Eur. J. Neurosci. 29, 1348-1356 (2009).

18. Fleck, M. W., Palmer, A. M. \& Barrionuevo, G. Potassium-induced long-term potentiation in rat hippocampal slices. Brain Res. 580, 100-105 (1992).

19. Baron, C., Benes, C., Van Tan, H., Fagard, R. \& Roisin, M. P. Potassium chloride pulse enhances mitogen-activated protein kinase activity in rat hippocampal slices. J. Neurochem. 66, 1005-1010 (1996).

20. Saitoh, H. \& Hinchey, J. Functional heterogeneity of small ubiquitin-related protein modifiers SUMO-1 versus SUMO-2/3. J. Biol. Chem. 275, 6252-6258 (2000).

21. Johnson, E. S. \& Gupta, A. A. An E3-like factor that promotes SUMO conjugation to the yeast septins. Cell 106, 735-744 (2001).

22. Tatham, M. H. et al. RNF4 is a poly-SUMO-specific E3 ubiquitin ligase required for arsenic-induced PML degradation. Nat. Cell Biol. 10, 538-546 (2008).

23. Tatham, M. H. et al. Polymeric chains of SUMO-2 and SUMO- 3 are conjugated to protein substrates by SAE1/SAE2 and Ubc9. J. Biol. Chem. 276, 35368-35374 (2001).

24. Lopantsev, V., Both, M. \& Draguhn, A. Rapid plasticity at inhibitory and excitatory synapses in the hippocampus induced by ictal epileptiform discharges. Eur. J. Neurosci. 29, 1153-1164 (2009).

25. Ludvig, N., Chao, K., Altura, B. T., Altura, B. M. \& Fox, S. E. Manipulation of pyramidal cell firing in the hippocampus of freely behaving rats by local application of K+ via microdialysis. Hippocampus 6, 97-108 (1996).

26. Craig, T. J. et al. Homeostatic synaptic scaling is regulated by protein SUMOylation. J. Biol. Chem. (2012).

27. Gong, B. et al. Ubiquitin hydrolase Uch-L1 rescues beta-amyloid-induced decreases in synaptic function and contextual memory. Cell 126, 775-788 (2006).

28. Gump, J. M. \& Dowdy, S. F. TAT transduction: the molecular mechanism and therapeutic prospects. Trends Mol. Med. 13, 443-448 (2007).

29. Gong, L., Kamitani, T., Fujise, K., Caskey, L. S. \& Yeh, E. T. Preferential interaction of sentrin with a ubiquitin-conjugating enzyme, Ubc9. J. Biol. Chem. 272, 28198-28201 (1997).

30. Xhemalce, B. et al. Role of SUMO in the dynamics of telomere maintenance in fission yeast. Proc Natl Acad Sci U S A 104, 893-898 (2007).

31. Buschmann, T., Lerner, D., Lee, C. G. \& Ronai, Z. The Mdm-2 amino terminus is required for Mdm2 binding and SUMO-1 conjugation by the E2 SUMO-1 conjugating enzyme Ubc9. J. Biol. Chem. 276, 40389-40395 (2001).

32. Mukhopadhyay, D. \& Dasso, M. Modification in reverse: the SUMO proteases. Trends Biochem. Sci. 32, 286-295 (2007).

33. Bailey, D. \& O'Hare, P. Characterization of the localization and proteolytic activity of the SUMO-specific protease, SENP1. J. Biol. Chem. 279, 692-703 (2004).

34. Barad, M., Bourtchouladze, R., Winder, D. G., Golan, H. \& Kandel, E. Rolipram, a type IV-specific phosphodiesterase inhibitor, facilitates the establishment of longlasting long-term potentiation and improves memory. Proc Natl Acad Sci U S A 95, 15020-15025 (1998).

35. Lambert, M. P. et al. Diffusible, nonfibrillar ligands derived from Abeta1-42 are potent central nervous system neurotoxins. Proc Natl Acad Sci U S A 95, 6448-6453 (1998).

36. Busciglio, J., Lorenzo, A., Yeh, J. \& Yankner, B. A. beta-amyloid fibrils induce tau phosphorylation and loss of microtubule binding. Neuron 14, 879-888 (1995).

37. Williamson, R. et al. Rapid tyrosine phosphorylation of neuronal proteins including tau and focal adhesion kinase in response to amyloid-beta peptide exposure: involvement of Src family protein kinases. J. Neurosci. 22, 10-20 (2002)

38. Hsiao, K. et al. Correlative memory deficits, Abeta elevation, and amyloid plaques in transgenic mice. Science 274, 99-102 (1996).

39. Westerman, M. A. et al. The relationship between Abeta and memory in the Tg2576 mouse model of Alzheimer's disease. J. Neurosci. 22, 1858-1867 (2002).

40. Barnes, P. \& Good, M. Impaired Pavlovian cued fear conditioning in Tg2576 mice expressing a human mutant amyloid precursor protein gene. Behav. Brain Res. 157, 107-117 (2005).

41. Li, Y. et al. Positive and negative regulation of APP amyloidogenesis by sumoylation. Proc Natl Acad Sci U S A 100, 259-264 (2003).

42. Dorval, V., Mazzella, M. J., Mathews, P. M., Hay, R. T. \& Fraser, P. E. Modulation of Abeta generation by small ubiquitin-like modifiers does not require conjugation to target proteins. Biochem. J. 404, 309-316 (2007).

43. Zhang, Y. Q. \& Sarge, K. D. Sumoylation of amyloid precursor protein negatively regulates Abeta aggregate levels. Biochem. Biophys. Res. Commun. 374, 673-678 (2008).

44. Haass, C. \& Selkoe, D. J. Soluble protein oligomers in neurodegeneration: lessons from the Alzheimer's amyloid beta-peptide. Nat. Rev. Mol. Cell Biol. 8, 101-112 (2007).

45. Loriol, C., Khayachi, A., Poupon, G., Gwizdek, C. \& Martin, S. Activity-dependent regulation of the sumoylation machinery in rat hippocampal neurons. Biol. Cell $105,30-45$ (2013).

46. Jaafari, N. et al. SUMOylation Is Required for Glycine-Induced Increases in AMPA Receptor Surface Expression (ChemLTP) in Hippocampal Neurons. PLoS One 8, e52345 (2013). 
47. Rosas-Acosta, G., Russell, W. K., Deyrieux, A., Russell, D. H. \& Wilson, V. G. A universal strategy for proteomic studies of SUMO and other ubiquitin-like modifiers. Mol. Cell. Proteomics 4, 56-72 (2005).

48. Vertegaal, A. C. et al. Distinct and overlapping sets of SUMO-1 and SUMO-2 target proteins revealed by quantitative proteomics. Mol. Cell. Proteomics 5, 2298-2310 (2006)

49. Ayaydin, F. \& Dasso, M. Distinct in vivo dynamics of vertebrate SUMO paralogues. Mol Biol Cell 15, 5208-5218 (2004)

50. Shen, L. N., Geoffroy, M. C., Jaffray, E. G. \& Hay, R. T. Characterization of SENP7, a SUMO-2/3-specific isopeptidase. Biochem. J. 421, 223-230 (2009).

51. Klann, E., Chen, S. J. \& Sweatt, J. D. Persistent protein kinase activation in the maintenance phase of long-term potentiation. J. Biol. Chem. 266, 24253-24256 (1991).

52. Klann, E., Chen, S. J. \& Sweatt, J. D. Increased phosphorylation of a $17-\mathrm{kDa}$ protein kinase C substrate (P17) in long-term potentiation. J. Neurochem. 58, 1576-1579 (1992).

53. Siciliano, J. C., Gelman, M. \& Girault, J. A. Depolarization and neurotransmitters increase neuronal protein tyrosine phosphorylation. J. Neurochem. 62, 950-959 (1994)

54. Rosenblum, K., Dudai, Y. \& Richter-Levin, G. Long-term potentiation increases tyrosine phosphorylation of the $\mathrm{N}$-methyl-D-aspartate receptor subunit $2 \mathrm{~B}$ in rat dentate gyrus in vivo. Proc Natl Acad Sci U S A 93, 10457-10460 (1996).

55. Rostas, J. A. et al. Enhanced tyrosine phosphorylation of the $2 \mathrm{~B}$ subunit of the Nmethyl-D-aspartate receptor in long-term potentiation. Proc Natl Acad Sci U S A 93, 10452-10456 (1996).

56. Akers, R. F., Lovinger, D. M., Colley, P. A., Linden, D. J. \& Routtenberg, A. Translocation of protein kinase $\mathrm{C}$ activity may mediate hippocampal long-term potentiation. Science 231, 587-589 (1986).

57. Sweatt, J. D. \& Kandel, E. R. Persistent and transcriptionally-dependent increase in protein phosphorylation in long-term facilitation of Aplysia sensory neurons. Nature 339, 51-54 (1989).

58. Lovinger, D. M., Wong, K. L., Murakami, K. \& Routtenberg, A. Protein kinase C inhibitors eliminate hippocampal long-term potentiation. Brain Res. 436, 177-183 (1987).

59. Malinow, R., Madison, D. V. \& Tsien, R. W. Persistent protein kinase activity underlying long-term potentiation. Nature 335, 820-824 (1988).

60. Malenka, R. C. et al. An essential role for postsynaptic calmodulin and protein kinase activity in long-term potentiation. Nature 340, 554-557 (1989).

61. Schafe, G. E., Nadel, N. V., Sullivan, G. M., Harris, A. \& LeDoux, J. E. Memory consolidation for contextual and auditory fear conditioning is dependent on protein synthesis, PKA, and MAP kinase. Learn Mem 6, 97-110 (1999).

62. Blum, S., Moore, A. N., Adams, F. \& Dash, P. K. A mitogen-activated protein kinase cascade in the CA1/CA2 subfield of the dorsal hippocampus is essential for long-term spatial memory. J. Neurosci. 19, 3535-3544 (1999).

63. Chamberlain, S. E. et al. SUMOylation and phosphorylation of GluK2 regulate kainate receptor trafficking and synaptic plasticity. Nat. Neurosci. 15, 845-852 (2012).

64. Sarge, K. D. \& Park-Sarge, O. K. Sumoylation and human disease pathogenesis. Trends Biochem. Sci. 34, 200-205 (2009).

65. Steffan, J. S. et al. SUMO modification of Huntingtin and Huntington's disease pathology. Science 304, 100-104 (2004).

66. McMillan, L. E., Brown, J. T., Henley, J. M. \& Cimarosti, H. Profiles of SUMO and ubiquitin conjugation in an Alzheimer's disease model. Neurosci. Lett. 502, 201-208 (2011).

67. Nistico, R. et al. Age-related changes of protein SUMOylation balance in the AbetaPP Tg2576 mouse model of Alzheimer's disease. Frontiers in pharmacology 5, 63 (2014).

68. Ahn, K. et al. Ubc9 gene polymorphisms and late-onset Alzheimer's disease in the Korean population: a genetic association study. Neurosci. Lett. 465, 272-275 (2009).

69. Grupe, A. et al. Evidence for novel susceptibility genes for late-onset Alzheimer's disease from a genome-wide association study of putative functional variants. Hum. Mol. Genet. 16, 865-873 (2007).
70. Corneveaux, J. J. et al. Association of CR1, CLU and PICALM with Alzheimer's disease in a cohort of clinically characterized and neuropathologically verified individuals. Hum. Mol. Genet. 19, 3295-3301 (2010).

71. Su, Y. F., Yang, T., Huang, H., Liu, L. F. \& Hwang, J. Phosphorylation of Ubc9 by Cdk1 enhances SUMOylation activity. PLoS One 7, e34250 (2012).

72. Vincent, I., Jicha, G., Rosado, M. \& Dickson, D. W. Aberrant expression of mitotic cdc2/cyclin B1 kinase in degenerating neurons of Alzheimer's disease brain. J. Neurosci. 17, 3588-3598 (1997).

73. Bowser, R. \& Smith, M. A. Cell cycle proteins in Alzheimer's disease: plenty of wheels but no cycle. J Alzheimers Dis 4, 249-254 (2002).

74. Dorval, V. \& Fraser, P. E. Small ubiquitin-like modifier (SUMO) modification of natively unfolded proteins tau and alpha-synuclein. J. Biol. Chem. 281, 9919-9924 (2006).

75. Lanz, T. A., Carter, D. B. \& Merchant, K. M. Dendritic spine loss in the hippocampus of young PDAPP and Tg2576 mice and its prevention by the ApoE2 genotype. Neurobiol Dis 13, 246-253 (2003).

76. Jacobsen, J. S. et al. Early-onset behavioral and synaptic deficits in a mouse model of Alzheimer's disease. Proc Natl Acad Sci U S A 103, 5161-5166 (2006).

77. Dong, H., Martin, M. V., Chambers, S. \& Csernansky, J. G. Spatial relationship between synapse loss and beta-amyloid deposition in Tg2576 mice. J. Comp. Neurol. 500, 311-321 (2007).

78. Dahlgren, K. N. et al. Oligomeric and fibrillar species of amyloid-beta peptides differentially affect neuronal viability. J. Biol. Chem. 277, 32046-32053 (2002).

79. Puzzo, D. et al. Picomolar amyloid-beta positively modulates synaptic plasticity and memory in hippocampus. J. Neurosci. 28, 14537-14545 (2008)

\section{Acknowledgments}

L.L. was the recipient of a F31 predoctoral fellowship from the NIH. This work was supported by NIH grant NS049442. We thank Dr. Olli Jänne (U. of Helsinki, Finland), Dr. Steven Dowdy (U. of California, San Diego) and Dr. Steve Goldstein (U. of Chicago) for plasmid gifts. We also thank members of the Arancio laboratory for critical discussion and feedback.

\section{Author contributions}

L.L., E.D. and O.A. conceived and designed the project. L.L., E.D., A.S., H.Z., F.S., M.F., I.O., F.M., N.A. and H.L. performed experiments and gathered data. L.L., E.D., A.S., H.Z., I.O., F.M. and O.A. analyzed data and prepared figures. M.S. and M.F. contributed reagents, materials and/or analysis tools. L.L. and O.A. wrote the manuscript. All authors reviewed the manuscript.

\section{Additional information}

Supplementary information accompanies this paper at http://www.nature.com/ scientificreports

Competing financial interests: The authors declare no competing financial interests.

How to cite this article: Lee, L. et al. Regulation of synaptic plasticity and cognition by SUMO in normal physiology and Alzheimer's disease. Sci. Rep. 4, 7190; DOI:10.1038/ srep07190 (2014).

This work is licensed under a Creative Commons Attribution-NonCommercialNoDerivs 4.0 International License. The images or other third party material in this article are included in the article's Creative Commons license, unless indicated otherwise in the credit line; if the material is not included under the Creative Commons license, users will need to obtain permission from the license holder in order to reproduce the material. To view a copy of this license, visit http:// creativecommons.org/licenses/by-nc-nd/4.0/ 


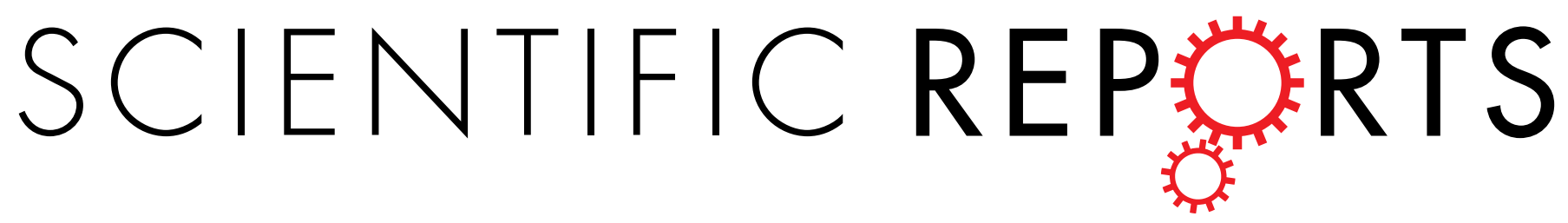

\section{Corrigendum: Regulation of synaptic plasticity and cognition by SUMO in normal physiology and Alzheimer's disease}

Linda Lee, Elena Dale, Agnes Staniszewski, Hong Zhang, Faisal Saeed, Mikako Sakurai, Mauro Fa', Ian Orozco, Francesco Michelassi, Nsikan Akpan, Helaina Lehrer \& Ottavio Arancio

Scientific Reports 4:7190; doi: 10.1038/srep07190; published online 2 December 2014; updated 07 July 2015

The original version of this Article contained a typographical error in the spelling of the author Helaina Lehrer which was incorrectly given as Helena Lehrer. This has now been corrected in the PDF and HTML versions of the Article. 